\title{
Bestrafen, Verwahren und danach Therapieren? - Das neue Therapie-Unterbringungsgesetz in der Kritik
}

Christine Morgenstern

Die Verfasserin geht davon aus, dass die nicht ganz offen ausgesprochenen Motive der Gesetzgebung, mit dem ThUG auch sog. Dissoziale Persönlichkeiten verwahren zu können, sich nicht EMRK-konform umsetzen lassen. Dissoziale können zwar als ,gefährlich" eingestuft werden, sind aber nicht notwendigerweise „krank". Zwar kann man ihnen eine Störung zuschreiben, aber diese weise in der Regel keinen „Krankheitswert“ auf und rechtfertige somit auch keinen Freiheitsentzug nach verbüßter Strafe.

\section{Ausgangslage}

Auch nach 18 Monaten sind die Konsequenzen des Urteils des Europäischen Gerichtshofs für Menschenrechte (EGMR) im Fall M. ./. Deutschland ${ }^{1}$ noch offen und die Diskussion ist nicht verstummt. ${ }^{2}$ Zwar sind zum 1.1.2011 das „Gesetz zur Neuordnung des Rechts der Sicherungsverwahrung und zu begleitenden Regelungen “ in Kraft getreten, das neben Änderungen der Vorschriften der Sicherungsverwahrung und der Führungsaufsicht auch das „Gesetz zur Therapierung und Unterbringung psychisch gestörter Gewalttäter" (ThUG, BGBl. I S. 2300, 2305) enthält. Mit ihm will der Gesetzgeber einerseits den völkerrechtsgemäßen Zustand herstellen und so seiner Umsetzungspflicht aus Art. 46 I EMRK folgen, andererseits aber so weit als möglich verhindern, dass die als sog. „Parallelfälle“ betroffene Gruppe von Sicherungsverwahrten, d. h. diejenigen, bei denen die Sicherungsverwahrung bereits vor 1998 angeordnet worden und inzwischen die damals geltende Höchstdauer erreicht ist, wirklich in die Freiheit entlassen werden.

Nach dem Beschluss des 5. Strafsenats des BGH vom 9.11.20103 schien zwar die Dringlichkeit zur Schaffung einer neuen rechtlichen Grundlage für die weitere Unterbringung der meisten mittelbar vom Straßburger Urteil Betroffenen (vorerst) weggefallen, weil das Gericht die Fortdauer der Sicherungsverwahrung zumindest in den schwersten Fällen weiterhin für zulässig hält. Damit können weiterhin als sehr gefährlich eingeschätzte Gewalt- und Sexualstraftäter nicht entlassen werden; selbst wenn das für sie zuständige OLG grundsätzlich aus dem Urteil des EGMR eine Pflicht zur Entlassung gefolgert hatte. Der 5. Strafsenat konnte insofern (zunächst) das letzte Wort sprechen, als er nach der (als erste gesetzgeberische Behelfsmaßnahme in Reaktion auf das Straßburger Urteil neugeschaffene) Divergenzvorlagepflicht nach $\$ 121$ II Nr. 3 GVG für die Vereinheitlichung der OLG-Rechtsprechung zuständig ist. Es geht dabei konkret um die Frage, ob das Urteil des EGMR die deutschen Gerichte dazu zwingt, in den relevanten Fällen die Maßregel nach zehnjährigem Vollzug für erledigt zu erklären. Er argumentierte in seinem Beschluss, dass der ausdrückliche, im Gesetzgebungsverfahren von 1998 geäußerte Wille des Gesetzgebers ihn an einer konventionskonformen Rechtsanwendung ${ }^{4}$ hindere.

Trotz dieses „Entlassungsmoratoriums“ bis zur endgültigen rechtlichen Klärung der Frage sah sich der Bundesgesetzgeber offenbar zum schnellen Handeln genötigt, weil er aufgrund hysterischer Medienberichte über die vor der genannten Entscheidung des 5. Strafsenats bereits Entlassenen ebenso wie politisch-populistischer Äußerungen einiger Landespolitiker ${ }^{5}$ unter Druck geraten war. Zudem stellt die für erforderlich gehaltene ständige polizeiliche Überwachung der solchermaßen Entlassenen ein erhebliches logistisches, finanzielles und rechtliches ${ }^{6}$ Problem dar. Hinzu kommt, dass auch gutwillige Justizverwaltungen bzw. Führungsaufsichtsstellen aufgrund des öffentlichen/ medialen Drucks erhebliche Probleme ${ }^{7}$ mit der Unterbringung der Entlassenen in Übergangseinrichtungen o. ä. hatten (bzw. noch haben). Während in Deutschland über das Reformpaket gestritten wurde, ergingen in Straßburg drei weitere Urteile, in denen Deutschland in gleich oder ähnlich gelagerten Fällen im Zusammenhang mit der Rückwirkungsproblematik bei Sicherungsverwahrten verurteilt wurde. ${ }^{8}$

\section{Die Regelungen des ThuG und ihre rechtlichen und praktischen Probleme}

\section{a) Die Rahmenbedingungen}

Noch immer herrscht innerhalb der höchsten deutschen Gerichte Uneinigkeit, wie rechtlich mit dem Urteil aus Straßburg umzugehen ist (dazu näher unten unter 3.). Abgesehen von den wenigen Fällen, in denen die Betroffenen vor dem Machtwort des 5. Strafsenats freigelassen worden sind, ist also nach einer endgültigen Klärung eine weitere Entlassungswelle zumindest denkbar. Hier sieht sich der Bundesgesetzgeber nun durch das ThuG gerüstet, die „Parallelfälle“ nach ihrer Entlassung aus dem Strafvollzug erneut - diesmal „therapeutisch“ - zu inhaftieren. Nach $\mathbb{1} 1$ des ThUG ist es anwendbar auf „eine wegen einer Straftat der in $\mathbb{S} 66$ Abs. 3 S. 1 StGB genannten Art verurteilte Person“, die „deshalb nicht länger in der Sicherungsverwahrung untergebracht werden kann, weil ein Verbot rückwirkender Verschärfungen im Recht der Sicherungsverwahrung zu berücksichtigen ist“. Solche Personen sollen ,in einer geeigneten geschlossenen Einrichtung “ untergebracht werden können. Die grundsätzlich erfasste Gruppe ist wohl etwas größer als zunächst angenommen: Medienberichten zufolge ${ }^{9}$ sind schon jetzt rund 120 Personen betroffen, weitere folgen nach und nach (bis ca. 2018, z. B. in Berlin ${ }^{10}$ ).

Die materiellen Voraussetzungen der Unterbringung sind neben der vorangegangenen Sicherungsverwahrung bzw. der „Altfalleigenschaft" das Vorliegen einer psychischen Störung und eine daraus resultierende hohe Wahrscheinlichkeit, dass eine schwere Gewalt- oder Sexualstraftat durch den Täter bevorsteht. Nimmt man die beiden letztgenannten Voraussetzungen ernst, so ergeben sich starke Ähnlichkeiten mit den Psychiatrie- bzw. Unterbringungsgesetzen der Länder, in denen die Unterbringung von psychisch Kranken in einem psychiatrischen Krankenhaus geregelt ist, „wenn und solange durch ihr krankhaftes Verhalten gegen sich oder andere eine gegenwärtige erhebliche Gefahr einer Selbstschädigung oder für die öffentliche Sicherheit besteht, die nicht 
anders abgewendet werden kann $[\ldots]^{\text {“ }}(\mathrm{zB}$ \11 I PsychKG M-V). Diese existierenden öffentlich-rechtlichen, präventiv ausgerichteten Unterbringungsgesetze, die dem Schutz und der Behandlung der betroffenen Kranken, jedoch ebenso dem Schutz Dritter bzw. der Öffentlichkeit dienen, sind Ländersache $^{11}$ - inwiefern hier zusätzlicher Regelungsbedarf auf Bundesebene besteht und daraus auch eine Bundeskompetenz konstruiert werden kann, hängt von der übrigen rechtlichen Bewertung $a b$.

Die Einrichtungen jedenfalls sollen - um eine vom EGMR angeprangerten Nähe zum Strafvollzug zu vermeiden - allein „medizinisch-therapeutisch“ sein ( $\$ 2$ ThuG). Das gerichtliche Verfahren ist aber zivilrechtlich (entnommen aus dem Gesetz über das Verfahren in Familiensachen und in den Angelegenheiten der freiwilligen Gerichtsbarkeit - FamFG), und eine Zivilkammer des Landgerichts ist zuständig. Hiermit eröffnet sich zumindest die Möglichkeit, dass neue Sichtweisen auf (ehemals) strafvollstreckungsrechtliche Sachverhalte, bei denen sich alle Akteure gut bekannt waren, eröffnen. Das für die praktische Umsetzung wahrscheinlich bedeutsamste Problem des ThUG ist damit auch angesprochen: Es fehlt an den nötigen Strukturen: Fraglich ist, ob genügend geeignete Gutachter zur Verfügung stehen, nötig sind außerdem geeignete Einrichtungen und schließlich ausreichend geeignetes Personal. Ganz klar hat der Bund hier den schwarzen Peter an die Länder weitergegeben (fairerweise muss man sagen, dass von einigen der Länderjustizminister, namentlich aus Bayern und Niedersachsen, auch die schärfsten Forderungen gekommen waren $\left.^{12}\right)$. Der Diskussionsprozess in Bezug auf die Standorte der geplanten Einrichtungen ist für die Öffentlichkeit nur teilweise nach zu verfolgen, lässt aber schon jetzt die Schwierigkeiten erkennen: In der Debatte waren bislang (ohne Anspruch auf Vollständigkeit) eine ehemalige Jugendarrestanstalt (Wiesloch in Baden-Württemberg), ein psychiatrisches Zentrum (Emmendingen in Baden-Württemberg), eine Station in einem Untersuchungshaftvollzugskrankenhaus (Hamburg), eine (dann ehemalige) Justizvollzugsanstalt (Oberhausen). Inwieweit die Umwandlung ehemaliger Justizvollzugsanstalten in medizinisch-therapeutische Einrichtungen möglich ist, ist sehr fraglich. Nach der Rechtsprechung des EGMR ist jedenfalls für die Rechtmäßigkeit einer Unterbringung nach Art. 5 I lit. e EMRK erforderlich, dass sie in einem Krankenhaus oder einer anderen angemessenen Einrichtung vollzogen wird, wobei es auf eine (materielle und personelle) Ausstattung ankommt, die tatsächlich eine Behandlung der psychischen Erkrankung ermöglicht. ${ }^{13}$ Von diesen rechtlichen Bedenken in Bezug auf die Eignung ehemaliger Justizvollzugseinrichtungen abgesehen, befürchten aber natürlich die Betreiber psychiatrischer Krankenhäuser oder anderer Behandlungseinrichtungen, dass ihre „zivilen“ psychiatrischen Patienten durch die Unterbringung ehemals Sicherungsverwahrter erneut stigmatisiert werden. ${ }^{14}$

\section{b) Die Betroffenen}

Was die Adressaten der neuartigen Unterbringung betrifft, ist das technische Kriterium des Altfalls klar - weitaus problematischer aber ist der in der Sache gewählte Anknüpfungspunkt der „psychischen Störung “ der Unterzubringenden. Dabei löst man sich vom Begriff des „psychisch Kranken“, wie er in der Regel in den genannten Unterbringungsgesetzen verwendet wird. In der Tat hat in der Psychiatrie der Begriff der "psychischen Störung“ den der „psychischen Erkrankung“ weitgehend abgelöst, Hintergrund ist dabei vor allem das Bemühen um eine Entstigmatisierung der Betroffenen gewesen. Die Gesetzesbegründung führt in diesem Zusammenhang aus (BT-Drs. 17/3403, 85): „Letztlich deckt der Begriff der ,psychischen Störung ${ }^{6}$ ein breites Spektrum von Erscheinungsformen ab, von denen nur ein Teil in der psychiatrisch-forensischen Begutachtungspraxis bzw. auf einer normativen Ebene als psychische Erkrankung gewertet wird.“ Die PsychKG bzw. Unterbringungsgesetze der Länder schränken bei ihren Unterbringungsvoraussetzungen (mit gutem Grund!) sämtlich das Eingangskriterium der Krankheit oder Störung dahingehend ein, dass sie „in einem erheblichen Ausmaß“ (z. B. $\$ 1$ II UBG BW) vorkommen müssen, bzw. qualifizieren sie inhaltlich: „Psychisch Kranke im Sinne diese Gesetzes sind Personen, die an einer Psychose, einer psychischen Störung, die in ihrer Auswirkung einer Psychose gleichkommt, oder einer mit dem Verlust der Selbstkontrolle einhergehenden Abhängigkeit von Suchtstoffen leiden“ ( $\mathbb{1} 1$ II PsychKG MV). Hervorzuheben ist weiterhin, dass die in ICD-10 und DSM-IV kodierten Diagnosen, die der Gesetzgeber in seiner Gesetzesbegründung heranzieht, für den forensischgutachterlichen Gebrauch allein nicht hinreichend verwendbar sind, sondern in einem klinisch-normativen Gesamtzusammenhang erst gewürdigt werden müssen. ${ }^{15}$ In diesem
Zusammenhang berichtet die Presse davon, dass es beim Bundesverfassungsgericht (hierzu sogleich) für ein gewisses Stirnrunzeln gesorgt hat, dass der Vertreter des Bundesjustizministeriums nicht genauer erklären konnte, wie der Begriff der „psychischen Störung " nun genau zu verstehen ist. ${ }^{16}$

Die weiteren materiellen Kriterien des ThUG dürften zwar durchaus die leichtfertige Anordnung einer Therapieunterbringung grundsätzlich (und richtigerweise) erschweren: Gefordert wird ein kausaler Zusammenhang zwischen der Störung und einer erheblichen Gefährlichkeit für Leib, Leben oder sexuelle Selbstbestimmung Dritter; außerdem muss eine „hohe Wahrscheinlichkeit" eines solchen Schadenseintritts vorliegen - ein Wahrscheinlichkeitsgrad, der nicht so ohne weiteres zu prognostizieren ist. Warum dann aber der Gebrauch des Begriffs der „psychischen Störung“? Er ist aus forensischer Sicht unzureichend und aus verfassungsrechtlicher Sicht mangels hinreichender Kriterien (s. o.) zur Eingrenzung zu unbestimmt. Festzuhalten ist: Die in der Begründung des ThuG genannte „dissoziale Persönlichkeitsstörung “ ist zwar eine anerkannte, wenngleich in ihrer jeweiligen konkreten Tragweite bzw. Krankheitswertigkeit zu differenzierende psychische Störung, ${ }^{17}$ sie erfasst aber nur eine Teilmenge der dissozialen Persönlichkeiten ${ }^{18}$ - sie jedoch möchte man mit der Formulierung ganz offenbar ${ }^{19}$ gerne erfassen.

\section{c) Vereinbarkeit mit der EMRK}

Damit stellt sich weitergehend die Frage, inwiefern die avisierte Personengruppe unter Art. 5 I lit. e EMRK fällt, bzw. ob die Unterbringung der Parallelfälle über das ThUG nach der EMRK gerechtfertigt sein kann (oder ob die nächsten Verurteilungen ins Haus stehen). Art. $5 \mathrm{I}$ lit e ist einer der in der EMRK abschließend aufgezählten Haftgründe und erlaubt eine Inhaftierung von „psychisch Kranken“ (in der deutschen Übersetzung; „persons of an unsound mind“ in der englischen, „un aliené“ in der französischen Originalfassung). Die Landgerichte, die nunmehr die ersten Anträge zu prüfen haben (siehe sogleich unten), müssen die EMRK (als einfaches Bundesrecht) in ihrer Auslegung durch den EGMR bei der Anwendung des Begriffs der „psychischen Störung “ beachten. Dabei ist es schwierig, anhand der EGMR-Rechtsprechung eine genaue Reichweite der Norm zu ermitteln, denn der Gerichtshof hat sich hierzu nie im Sinne einer Definition geäußert, sondern 
lediglich Leitkriterien (Winterwerp ./. Niederlande, 24.10.1979, Rn. 39.) entwickelt. Erforderlich ist danach, dass verlässlich nachgewiesen sein muss, dass eine Geisteskrankheit vorliegt, für die Unterbringung eine Notwendigkeit besteht und sie nur so lange dauern darf, wie die Krankheit und die daraus resultierende Notwendigkeit der Unterbringung fortbestehen - was regelmäßig überprüft werden muss. Was eine psychische Erkrankung ist, will der Gerichtshof selbst nicht definieren, weil das medizinische Verständnis dieses Konzepts sich ständig fortentwickelt. Es ist aber festzustellen, dass der EGMR unter Art. 5 I lit. e stets Fälle subsumiert, in denen nicht irgendwelche, sondern erhebliche psychische Störungen (d. h. mit Krankheitswert) vorlagen. Gesprochen wird in der Regel von einer „serious“ oder „true“ mental disorder" (Schummer ./. Deutschland, Rn. 56 bzw. Haidn /. Deutschland, Rn. 56). Im Fall M. ./. Deutschland wurde dem Antragsteller im Vollstreckungsverfahren jedoch gerade bescheinigt, keine im Rahmen

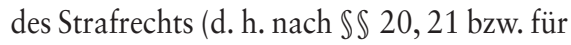
$\$ 63 \mathrm{StGB}$ ) relevante psychische Erkrankung mehr aufzuweisen. Im Parallelfall Kallweit ./. Deutschland wurde vom Tatgericht und im Vollstreckungsverfahren immer wieder festgehalten, dass der Antragsteller unter einer dissozialen Persönlichkeitsstörung leide, allerdings wurde daraus nicht geschlossen, dass seine Schuldfähigkeit vermindert oder aufgehoben sei oder dass die Sicherungsverwahrung in einem psychiatrischen Krankenhaus zu vollstrecken sei. Für den EGMR folgt daraus, dass die psychische Beeinträchtigung in diesem Fall nicht die für eine Unterbringung nach Art. 5 I lit. e EMRK erforderliche Schwere aufweist. Inwieweit eine solche weitgehende Gleichsetzung mit der Erheblichkeit der in $\mathbb{S} 20,21$ StGB vorausgesetzten Störungen tatsächlich gerechtfertigt ist, ist fraglich. Für den hier interessierenden Problemkreis bedeutet es, dass nach Auffassung des EGMR die meisten der Parallelfälle wohl aus einem nach Art. 5 I lit. e zu rechtfertigenden Bereich herausfallen: Entweder es liegt schon gar keine klassifizierbare psychische Erkrankung vor (sondern „nur" eine gefährliche, dissoziale Persönlichkeit) oder die psychische Störung weist mangels Krankheitswert keine Schwere auf, die nach den Kriterien des EGMR Bestand haben würde. Nochmals: Die Gefährlichkeit alleine genügt nicht!

Sollten neue Gutachter dennoch psychische Erkrankungen und eine daraus resultierende, für das ThuG ausreichende Gefährlichkeit attestieren, schließen sich auch im
Hinblick auf deutsches Verfassungsrecht (zumindest) zwei Fragen an: Zum Ersten: Warum ist während der Sicherungsverwahrung keine Therapie erfolgt bzw. warum sind sie nicht $z$. B. nach $\$ 67$ a II StGB in die Psychiatrie überstellt worden ${ }^{20}$ Sollte die Antwort gewesen sein: „Weil sie nicht therapiefähig sind.“ dann dürfte eine „therapeutische Unterbringung “ (nimmt man diese Bezeichnung ernst) wohl kaum in Frage kommen. Rechtlich betrachtet, geht es dann um die „Geeignetheit“ des Eingriffs, also der Unterbringung. Das Bundesverfassungsgericht ${ }^{21}$ hat zur Frage der Behandlungsunterbringung nach $\ 64$ StGB schon ausgeführt, dass therapeutische Eingriffe, die ja definitionsgemäß auf eine Besserung der Situation hinwirken sollen, nur möglich sind, wenn eine solche auch erreichbar erscheint; diese Argumentation dürfte auch hier tragen. Zum Zweiten: Sollte die Antwort anders ausfallen, also (mittlerweile) Therapiefähigkeit oder -bereitschaft festzustellen sein, warum kommt dann bei festzustellender Gefährlichkeit - als milderes Mittel - keine Unterbringung nach PsychKG in Betracht? Rechtlich betrachtet geht es nun um die Erforderlichkeit. Es liegt auf der Hand, dass Praktikabilitätserwägungen zunächst dagegen sprechen - keine reguläre Psychiatrie wird auf ihrer geschlossenen Station gerne jemanden aufnehmen, der jahrzehntelange Knasterfahrung hat und möglicherweise als "Systemsprenger" gilt. Dennoch ist zu bedenken, dass die Garantien für die Freiheitsrechte, die die PsychKG geben, insbesondere im Hinblick auf Dauer der Unterbringung bzw. die Möglichkeit, jederzeit einen Antrag auf Überprüfung zu stellen, weitaus größer sind als diejenigen, die nach dem ThuG nun zu Geltung kommen sollen. Rechtlich sind die Betroffenen aber prinzipiell in derselben Situation: Eine Strafe ist nicht (mehr) zu vollstrecken, ein Delikt hat es (noch) nicht gegeben. Interessanterweise hat der EGMR im Fall Haidn ausdrücklich kritisiert, dass niemals auch nur geprüft worden ist, den Betroffenen statt nach dem neugeschaffenen Bayerischen Straftäter-Unterbringungsgesetz nach dem „zivilen“ psychiatrischen Unterbringungsgesetz zu behandeln (vgl. Haidn ./. Deutschland, Rn. 92).

Einen Verstoß gegen Art. 5 I EMRK stellt im Übrigen für sich genommen auch $\mathbb{} 14$ ThUG dar, der im Rahmen der einstweiligen Anordnung eine vorläufige Unterbringung erlaubt, eine psychiatrische Begutachtung zur Feststellung aber erst nach drei Monaten zwingend notwendig macht. Eine solche einstweilige Unterbringung soll mög- lich sein, wenn „Gründe für die Annahme bestehen, dass die Voraussetzungen für die Anordnung einer Therapieunterbringung gegeben sind“ und ein „dringendes Bedürfnis zum sofortigen Tätigwerden“ besteht. Dass diejenigen Kommunen (die als untere Verwaltungsbehörde nach $\$ 5$ ThUG Antragsteller sein können), die Polizeikräfte zur Rundumüberwachung der Entlassenen abstellen müssen, ein solches „Bedürfnis“ verspüren, ist nachvollziehbar - ein anerkannter Haftgrund ist es nicht. Eine weitere übergangsweise Unterbringung kann außerdem dann, wenn die Gutachten aufgrund bestimmter Schwierigkeiten nicht schneller erstellt werden können, bis zu zwölf Monate verlängert werden. Wahrscheinlich wollte der Gesetzgeber hier realistisch sein und einem Engpaß in Bezug auf die verfügbaren forensischen Gutachter vorbauen, eine derartig ausgedehnte vorläufige Inhaftierung ist jedoch vor dem Hintergrund der EMRK nicht haltbar. ${ }^{22}$

\section{Aktuelle Entwicklungen und Ausblick}

Abschließende Entscheidungen aus Karlsruhe werden erst in den nächsten Monaten erwartet: Das Bundesverfassungsgericht hat über die Frage der Umsetzung der Urteile aus Straßburg im Februar 2011 verhandelt, ${ }^{23}$ eine Entscheidung ist wohl im Sommer zu erwarten. Die vom 5. Senat im o. g. Anfragebeschluss gestellte Frage, ob nämlich der 4. Senat an seiner Auffassung, das Urteil aus Straßburg ergebe die Notwendigkeit $\mathbb{2}$ VI StGB in einer die Rückwirkung hindernden Weise auszulegen, ${ }^{24}$ festhalte, ist unterschiedlich beantwortet worden: Der 5. Senat selbst, der 1. und der 2. Senat widersprechen der Auffassung des 4. Senats; der 3. Senat folgt ihm. ${ }^{25}$ Der Große Senat wird daher nun nach $\$ 132$ III GVG entscheiden müssen. Bis dahin liegen alle weiteren Entscheidungen auf Eis, bis zur Erledigung des Verfahrens werden die Akten an die vorlegenden Oberlandesgerichte zurückgegeben. Sie überprüfen nun nach den vom 5. Senat aufgestellten neuen Kriterien weiter nach $\$ 67 \mathrm{e}$ Abs. 1 Satz 1, $\$ 67 \mathrm{~d}$ Abs. 3 Satz 1, Abs. 2 StGB, inwiefern der Vollzug der Sicherungsverwahrung für erledigt erklärt oder ausgesetzt werden kann oder ob eine „hochgradige Gefahr schwerster Gewalt- und Sexualverbrechen aus konkreten Umständen in der Person oder dem Verhalten des Untergebrachten abzuleiten ist“ (Leitsatz des Vorlagebeschlusses vom 9.11.2010). 
Wie Gerichte mit dieser Situation umgehen, zeigt schön das Berliner Beispiel: Zwei Personen, die als „Parallelfälle“ einzuordnen sind, sind auf der Grundlage dieser Rechtsprechung unter strengsten Auflagen entlassen worden, weil eine solche hochgradige Gefahr eben nicht mehr attestiert werden konnte. In einem dritten Fall waren jedoch die Prognosen so schlecht, bzw. die Gefährlichkeit so hoch, dass das Kammergericht Berlin ${ }^{26}$ - obwohl es die Auffassung des 4. Strafsenats teilt und zum derzeitigen Schwebezustand harsche Worte der Kritik fand - über die sofortige Beschwerde des Betroffenen nicht entscheidet. Der Betroffene bleibt damit verwahrt.

Gleichzeitig wird in mehreren Zeitungen über „den ersten Fall“, in dem ein Antrag auf Therapieunterbringung verhandelt wird, berichtet. Die drei hier bekannten „ersten Fälle“ stammen aus Hamburg, ${ }^{27}$ Dortmund $^{28}$ und Freiburg, ${ }^{29}$ wo die zuständigen OLG mehrere Personen entlassen hatten bzw. wohin solchermaßen Entlassene gezogen waren. Das Freiburger Landgericht hat insofern ein (noch) größeres Problem als die beiden anderen Gerichte, als hier noch nicht einmal eine Einrichtung zur Verfügung steht, nachdem die bisherigen Vorschläge - Wiesloch und Emmendingen - offenbar am Widerstand der Bevölkerung (und, kurz vor der Wahl, am Widerstand der Abgeordneten des Wahlkreises?) gescheitert sind. Offenbar will man, dass nun die Gerichte Fakten schaffen - aus dem baden-württembergischen Sozialministerium hieß es, wenn das Gericht eine Therapieunterbringung anordne, werde das Land schon eine Einrichtung präsentieren können. ${ }^{30}$ Damit werden basale rechtliche Grundsätze nun endgültig auf den Kopf gestellt: Wenn Richter erst dafür sorgen müssen, dass die Voraussetzungen zur Anwendung von Gesetzen geschaffen werden, dürften sie sich - milde formuliert - vom Gesetzgeber nicht mehr ernst genommen fühlen. Ganz offenbar sind sie aber nicht bereit, das Schwarze-Peter-Spiel mitzuspielen. Im Freiburger wie auch im Dortmunder Fall wurden von Gerichtsprechern darauf hingewiesen, dass sich die Verfahren mehrere Monate hinziehen könnten. Und: Die oben kritisierte, Möglichkeit einer vorläufigen Unterbringung wurde offenbar nicht in Betracht gezogen. Das könnte ein Anlass zur Hoffnung sein, dass sich die Therapieunterbringung durch Zeitablauf erledigt - wer sich (selbst unter mehr oder weniger strengen Sicherheitsvorkehrungen) - über einen gewissen Zeitraum in Freiheit bewährt, dürfte noch schwerer wieder un- terzubringen sein; darauf hatte sogar die Gesetzesbegründung schon hingewiesen. Vielleicht verliert das Problem auch dadurch an Bedeutung, dass es den Führungsaufsichts- bzw. Polizeidienststellen (teilweise) besser gelingt, die Anonymität der betroffenen Entlassenen zu wahren und so den öffentlichen Druck soweit zu verringern, dass Anträge auf Unterbringung zumindest aus politischen Gründen nicht mehr gestellt werden. ${ }^{31}$ So könnte - abgesehen von den nicht zu behebenden rechtlichen Mängeln - das ThuG am Ende totes Recht sein, weil die Praxis es nicht nutzt.

Als Fazit bleibt: Eine menschenrechtskonforme Lösung, nach der für die zu Unrecht über die Zehn-Jahres-Frist hinaus verwahrten, noch als gefährlich begutachteten Straftäter zukünftig eine ,andere“ Unterbringung möglich ist, ist über die PsychKG der Länder hinaus nicht vorhanden. Darüber hinaus muss mit Sorge betrachtet werden, wie der Begriff der „psychischen Erkrankung “ jenseits (bzw. im Randbereich) der $\mathbb{S} \mathbb{S} 20,21$ StGB aufgeweicht wird und sich das Kriterium der Gefährlichkeit Raum schafft. Festzuhalten bleibt: Für Schuldfähige müssen die Garantien des Schuldstrafrechts gelten, psychisch kranke Straftäter müssen rechtzeitig Therapie bzw. ernsthafte Therapieangebote erhalten. Die Tatsache, dass unser Recht seit dem Wegfall der Höchstgrenze der Sicherungsverwahrung eine dritte Kategorie der „bloß Gefährlichen“, die gegebenenfalls für immer verwahrt werden müssen, kennt, ist zu beklagen. Dass denen, die unter Verstoß gegen das Rückwirkungsverbot über zehn Jahre hinaus verwahrt worden sind, nun weiterhin eine Zwangsunterbringung von unbestimmter Dauer droht, ist ein rechtsstaatliches Armutszeugnis.

Nach Drucklegung ist am 4.5.2011 die Entscheidung des BVerfG ergangen und sogleich auch veröffentlicht worden. Sie erklärt sämtliche Regelungen der Sicherungsverwahrung für verfassungswidrig und räumt dem Gesetzgeber zwei Jahre zur völligen Neukonzeption ein. Naturgemäß kann das komplexe Urteil hier nicht gewürdigt werden. Nur so viel: Für den BGH ergibt sich, dass der 5. Senat mit seiner Auffassung richtig lag; die vom EGMR-Urteil betroffenen Parallelfälle waren nach Ansicht des BVErfG nicht automatisch zu entlassen. Für den Gesetzgeber - nicht nur den aktuellen, sondern auch die vorherigen! - ist das Urteil eine strenge Rüge. Die vorgebliche „Neuordnung der Sicherungsverwahrung " vom Dezember 2010 hat die in der Entscheidung des Gerichts von 2004 deutlich formulierten Erfordernisse einer besonderen Konzeption der Maßregel unter der Berücksichtigung des Abstandsgebots nicht umgesetzt und ist deshalb verfassungswidrig. Die Regelungen, die rückwirkende Kraft entfalten, sind zwar nicht unter dem Aspekt des Art. 103 II GG, wohl aber unter dem Aspekt des Vertrauensschutzgebotes verfassungswidrig gewesen. Das hier interessierende ThuG schließlich ist nicht direkt von der Entscheidung betroffen, es bleibt in Kraft. Dennoch werden übergangsweise bis zum 31.5.2013 keine Parallelfälle entlassen, bei denen eine „hochgradige Gefahr schwerster Gewalt- und Sexualstraftaten" vorliegt, wenn die Betroffenen an einer dem ThuG entsprechenden psychischen Störung leiden. Ist dies nicht der Fall, sind sie zu entlassen - auch hier war das BVerfG aber großzügig: Dies muss erst zum Jahresende geschehen. Damit akzeptiert das Gericht gerade die hier problematisierte Regelung des ThuG und die neue Unterbringungsform und äußert nebenbei auch, dass es sie grundsätzlich für vereinbar mit Art. 5 EGMR hält. Die Begründung hierfür ist, dass „die Bestimmungen der Voraussetzungen einer psychischen Störung ...in erster Linie dem Gesetzgeber obliegt.“ Zur Frage, ob der Begriff der „Störung “ nicht zu grundsätzlich zu weit ist (wie hier vertreten), äußert sich das Gericht aber nicht.

\section{Literatur}

1. Harders I, Rohrbach M (2011) Führungsaufsicht im Aufwind? Bericht zur Fachtagung der DBH vom 4.-5.11.2010 in Kassel. BewHi 58 (im Erscheinen).

2. Heckmann, D. (2009) Juristische Grundlagen der öffentlich-rechtlichen Unterbringung. In: Kröber H-L, Dölling D, Leygraf N, Saß $\mathrm{H}$ (Hrsg) Handbuch der Psychiatrie, Bd. 5. Steinkopff, Heidelberg, S 137-166.

3. Kinzig J (2010), Das Recht der Sicherungsverwahrung nach dem Urteil des EGMR in Sachen M. gegen Deutschland. NStZ 30: 233239.

4. Kinzig, J. (2011): Die Neuordnung des Rechts der Sicherungsverwahrung, S. 177-182.

5. Kreuzer, A. (2011): Neuordnung der Sicherungsverwahrung: Fragmentarisch und fragwürdig trotz sinnvoller Ansätze, Strafverteidiger, S. 122-131.

6. Laue, C. (2010): Die Sicherungsverwahrung auf dem europäischen Prüfstand, JR, S. 198204.

7. Müller, H.-E. (2010): Die Sicherungsverwahrung, das Grundgesetz und die Europäische Menschenrechtskonvention. Strafverteidiger, S. 207-212.

8. Saß, H. (2006) Biographie, Persönlichkeit und Verantwortung. In: Schneider, F. (Hrsg) Symposium anlässlich des 60. Geburtstags von Henning Saß. Springer, Heidelberg, S 395-404.

9. Saß, H., Habermeyer, E. (2007) Die Begutachtung von Persönlichkeitsstörungen aus psychopathologischer Sicht. ForensPsychiatrPsycholKriminol 1: 156-161. 


\section{Fußnoten}

$1 \mathrm{M}$. gegen Deutschland, Individualbeschwerde Nr. 19359/04, EuGRZ 2010, 25., hierzu z. B. Laue 2010, Müller 2010.

2 Z. B. Kinzig 2010 und 2011; Kreuzer 2011.

3 Beschlüsse 5 StR 394/10, 5 StR 440/10 u. 5 StR 474/10.

4 Es geht dabei darum, ob die EMRK in ihrer Auslegung durch den EGMR für die Maßregel der Unterbringung in der Sicherungsverwahrung eine die Rückwirkung generell hindernde "andere Bestimmung“ im Sinne des $\mathbb{} 2$ Abs. 6 StGB darstellt.

5 Pressemitteilungen der jeweiligen Justizministerien: Bayern: v. 11.5.2010 („EGMR-Urteil zur Sicherungsverwahrung rechtskräftig“), 13.7.2010 („Bundesverfassungsgericht lässt Sicherungsverwahrten in Haft"); Niedersachsen: Pressemitteilungen v. 12.5.2010 („Justizminister hält an Sicherungsverwahrung fest" ), 27.5.2010 (,Justizminister begrüßt Entscheidung des OLG Celle zur Sicherungsverwahrung“), 7.6.2010 („EGMR kann deutsche Sicherungsverwahrung nicht aufheben“).

6 Schon die gesetzliche Grundlage, die eine längerfristige Observation ermöglicht, ist umstritten; sie findet sich in speziellen Vorschriften der Landes-Polizeigesetzes oder notfalls in der polizeilichen Generalklausel. Bislang haben die Gerichte zumindest für eine „Übergangszeit“ die Rundum-Überwachung auch unter Berücksichtigung von Aspekten der Privatsphäre (selbst der von Dritten, vgl. LG Aachen, Urteil vom 24.1.2011 - 6 K 140/10), der informationellen Selbstbestimmung und der Verhältnismäßigkeit für (einstweilen) zulässig erklärt (z. B. VGH Mannheim, Prozesskostenhilfe-Verfahren, Beschlüsse vom 7.3.2011 (1 S 184/11 bis 186/11) OVG Saarlouis Beschluss vom 16.12.2010, 3 B 284/10)). Eine differenzierte Auffassung hat das OLG Brandenburg vertrete, vgl. Beschluss vom 21.2.2010, $11 \mathrm{Wx} 91 / 09$, und im konkreten Fall die Zulässigkeit einer Observation über sechs Monate abgelehnt.
7 Das zeigten deutlich Diskussionsbeiträge auf einer Fachtagung der DBH zur Führungsaufsicht, vgl. Harders/Rohrbach 2011.

8 Die sind die Fälle Kallweit, Mautes und Schummer, alle Urteile vom 13.1.2011. Der ebenfalls am 13.1.2011 entschiedene Fall Haidn liegt etwas anders; hier hatte sich der Antragsteller, der inzwischen nach $\$ 63$ StGB im Maßregelvollzug untergebracht ist, gegen seine nachträglich angeordnete Verwahrung nach dem vom BVerfG für verfassungswidrig erklärten, aber im Wege der Fortgeltungsanordnung für eine Übergangszeit wirksam gebliebene Bayerischen Straftäter-Unterbringungsgesetz gewandt. Alle Urteile sind unter www.echr.coe.int in der HUDOC-Datenbank zu finden.

9 Taz vom 7.1.2011, http://www.taz.de/1/politik/ deutschland/artikel/1/schleswig-holstein-hatbeste-loesung/. Ein regelmäßig aktualisierter Überblick findet sich unter www.strafvollzugsarchiv.de.

10 Tagesspiegel,3.3.2011,http://www.tagesspiegel. de/berlin/kontrolle-nach-dem-knast/3897890. html.

11 Das ist aus Art. 30, 70 GG herzuleiten - sowohl das Gesundheitsrecht als auch die Gefahrenabwehr sind Regelungsbereiche der Länder, hierzu Heckmann 2009

13 Ashingdane ./. Vereinigtes Königreich, Urt. v. 28.5.1985; Aerts ./. Belgien, Urt. 30.7.1998; Haidn ./. Deutschland, Urt. v. 13.1.2011; Kallweit ./. Deutschland, Urt. v. 13.1.2011.

14 So der Verband der bayerischen Bezirke in einem Brief an die bayerische Sozialministerin Haderthauer v. 19.11.2010, http://www.baybezirke.de.

$15 \mathrm{Vgl}$. auch Leygraf in seiner Stellungnahme. Alle Stellungnahmen zum Gesetzentwurf (BT-Drs. 17/3403) unter http://www.bundestag.de/bundestag/ausschuesse17/a06/anhoerungen/02 Sicherungsverwahrung/04_Stellungnahmen/index.html.
16 Bericht gefunden z. B. bei RP Online vom 8.2.2011, http://www.rp-online.de/politik/ deutschland/Karlsruher-Richter-tendieren-zumWegsperren_aid_962307.html.

$17 \mathrm{Saß} 2006,398$.

$18 \mathrm{Saß/Habermeyer} 2007$.

19 Offen ausgesprochen wurde das während der öffentlichen Anhörung vor dem Rechtsausschuss vom Sachverständigen Heuer (Prot. 28 des Rechtsausschusses vom 10.22.2010, S. 7).

20 So war bei dem Antragsteller in der Rechtssache vor dem EGMR Kallweit ./. Deutschland (Urt. d. EGMR v. 13.1.2011, Rn. 6-12) ein Verlegungsantrag von der Sicherungsverwahrung in ein psychiatrisches Krankenhaus nach $\mathbb{6} 67$ II StGB abgelehnt worden, vgl. hierzu auch Kinzig 2011, 182.

21 NStZ 1994, S. 578, vgl. hierzu auch die Stellungnahme von Wankel (oben Fn. 15).

$22 \mathrm{Zu}$ den streng limitierten Möglichkeiten einer vorläufigen Unterbringung zur weiteren Begutachtung nach Art. 5 I lit. c EMRK vgl. Eriksen ./. Norwegen, Urt. d. EGMR v. 27.51997..

$23 \mathrm{Vgl}$. hierzu den Magazinbeitrag von Sonnen in diesem Heft.

24 Beschluss vom 12.5.2010, NStZ 2010, 567.

251 ARs 22/10 vom 15.12.2010, 2 ARs 456/10 vom 22.12.2010, 4 ARs 27/10 vom 18.1.2011 und 3 AR 35/10 vom 17.2.2011.

262 Ws 642/10, Beschluss vom 3.3.2011.

27 Justizbehörde Hamburg, Pressemitteilung der Freien und Hansestadt Hamburg vom 13.2.2011.

28 Ruhrnachrichten vom 1.2.2011.

29 SüdwestPresse Online vom 18.2.2011. (http:// www.swp.de/ulm/nachrichten/suedwestumschau/Pilotverfahren-um-Sextaeter;art4319,84 6398).

30 Schwäbisches Tagblatt vom 26.1.2011.

31 Hinweise finden sich in Presseberichten aus Münster („Ex-Sicherungsverwahrte leben unerkannt", Westfälische Nachrichten vom 14.1.2011.

\section{Therapie-Unterbringung - aber wo?}

Ende März hat eine Zivilkammer des Landgerichts Freiburg entschieden, dass ein nach dem EGMR-Urteil entlassener ehemaliger Sicherungsverwahrter nicht nach dem Therapieunterbringungsgesetz (ThuG) untergebracht werden kann, weil die Stadt Freiburg als Antragstellerin nicht angeben konnte, wo die Unterbringung denn erfolgen sollte. Auch die dagegen gerichtete Beschwerde - die diesmal ein Gebäude auf dem Gelände der JVA Heilbronn als „geplantem“ und „vorläufigen“ Unterbringungsort angab - wurde abgewiesen. Dieses Gebäude dient nach Angaben der Badischen Zeitung mit seinen 10 Zellen dazu, Insassen in Zeiten der Überbelegung aufzunehmen. Das Gericht begründete seine Entscheidung laut einer Pressemitteilung vom 20.4.2011 damit, dass diese Art der Unterbringung nicht den gesetzlichen Anforderungen nach dem ThUG entspreche, „die ausdrücklich eine räumliche Trennung der Therapieeinrichtung von Einrichtungen des Strafvollzugs “ verlangen.

Die Bundesländer tun sich sehr schwer damit, geeignete Einrichtungen zu finden und sehen oftmals offenbar wenig Alternativen zu umgewidmeten Einrichtungen des Justizvollzugs: In Hamburg soll eine Station des Zentralkrankenhauses der Untersuchungshaftvollzugsanstalt zur Therapieunterbringung dienen; in Nordrhein-Westfalen ist es zumindest für die kommenden zwei Jahre die JVA Oberhausen. Die bayerische Sozialministerin Haderthauer hingegen will die mehr als 30 Sicherungsverwahrten, die in Bayern derzeit vom EGMR-Urteil betroffen sind bzw. sein werden, in der Forensisch-Psychiatrischen Bezirksklinik Straubing, deren Träger der Bezirk Niederbayern ist, unterbringen. Den bereits deutlich artikulierten Widerstand der bayerischen Bezirke will sie nach einem Bericht der Süddeutschen Zeitung mit Zugeständnissen an anderer Stelle überwinden. Ihre Kollegin Steffens aus Nordrhein-Westfalen hatte bereits im Januar 2011 in Bezug auf die Umwidmung der JVA Oberhausen betont, die Sicherheit der Bevölkerung stehe für sie an erster Stelle. In derselben Pressemitteilung wird auch die Forderung des Oberhausener Bürgermeisters, „Freigang“ der Untergebrachten könne es keinesfalls geben, zitiert.

Es wird deutlich: Die Länder haben sich in aller Eile auf die Suche nach gesetzeskonformen Standorten zur Umsetzung des ThuG gemacht - Widerstand regt sich überall, zufriedenstellende Konzepte gibt es bislang nicht. Soweit sie (Übergangs)lösungen gefunden haben, ist allenfalls der Unterbringungs- und hier vor allem der Sicherungsaspekt berücksichtigt. Über die Ausstattung der Unterbringungsstandorte, vor allem, welches Personal dort arbeiten - und therapieren! - soll, ist bislang nichts zu hören. 